HOUSEHOLD ENERGY STUDIES: THE GAP BETWEEN THEORY AND METHOD

by

Tracey Crosbie

\author{
Reprinted from \\ ENERGY \& \\ ENVIRONMENT \\ VOLUME 17 No. 52006
}




\title{
HOUSEHOLD ENERGY STUDIES: THE GAP BETWEEN THEORY AND METHOD
}

\author{
Tracey Crosbie \\ School of Environment and Development \\ University Of Manchester, Oxford Road, Manchester, M13 9PL, United Kingdom. \\ E-mailTracey.Crosbie@Manchester.ac.uk
}

\begin{abstract}
AFFILIATIONS
This work forms part of the Carbon Reduction in Buildings (CaRB) Consortium, which has 5 UK partners: De Montfort University, University College London, Reading University, Newcastle University and Sheffield University. They are supported by the Carbon Vision initiative, which is jointly funded by the Carbon Trust and the Engineering and Physical Sciences Research Council, with additional support from the Economic and Social Research Council and the Natural Environment Research Council. The partners are assisted by a steering panel of representatives from UK industry and government.
\end{abstract}

\begin{abstract}
At the level of theory it is now widely accepted that energy consumption patterns are a complex technical and socio-cultural phenomenon and to understand this phenomenon, it must be viewed from both engineering and social science perspectives. However, the methodological approaches taken in household energy studies lag behind the theoretical advances made in the last ten or fifteen years. The quantitative research methods traditionally used within the fields of building science, economics, and psychology continue to dominate household energy studies, while the qualitative ethnographic approaches to examining social and cultural phenomena traditionally used within anthropology and sociology are most frequently overlooked. This paper offers a critical review of the research methods used in household energy studies which illustrates the scope and limitations of both qualitative and quantitative research methods in this area of study. In doing so it demonstrates that qualitative research methods are essential to designing effective energy efficiency interventions.
\end{abstract}

Key words: Household energy studies, research methods, qualitative, quantitative 


\section{INTRODUCTION}

After more than thirty years of predominantly quantitative research exploring household energy conservation [1 page 13] household energy consumption continues to grow in many countries [2-6].Writing in 1992 Lutzenhiser argued that " $a$ widely accepted theoretical model centred upon physical systems and individual consumers has done a poor job of accounting for household energy use, and that this model is giving way to an approach which organizes the strengths of various disciplines in a cultural or lifestyle model of energy consumption"[7 page 49]. Whilst this may well be true at the theoretical level, the methodological approaches taken in household energy studies lag behind the theoretical advances made in the last ten or fifteen years. The quantitative research methods traditionally used within the fields of building science, economics, and psychology continue to dominate household energy studies, while the qualitative ethnographic approaches to examining social and cultural phenomena traditionally used within anthropology and sociology are most frequently overlooked. This is problematic because quantitative methods of data collection cannot supply the "finely grained and detailed information" necessary for thorough examinations of the socio-cultural influences on household energy consumption [8 page 52].

To reduce household energy consumption we need to find methods of encouraging the adoption and use of energy efficient technologies. Socially and culturally sensitive research into household energy consumption offers the potential to inform the introduction of technically proved technologies into appropriate social practices [9]. For example, qualitative research has found "the strong social significance of cosiness leads to overheating and over lighting as insurance against social failure" in Norwegian households [10 page 798], suggesting that low energy alternatives to producing the same cosy effect may provide at least part of the solution to reducing the energy used to light and heat Norwegian homes. However, as quantitative methods of data collection simply do not provide the depth of data necessary for unpacking the socio-cultural shaping of household energy consumption $[8,10,11]$, the continued dominance of these approaches within household energy studies limits the usefulness of this work in this regard.

This is not to suggest that quantitative methods of data collection have no place in household energy studies. The aim of this paper is to illustrate that quantitative methods of data collection must be successfully combined with more qualitative research methods, if we are to expand our understanding of the social and cultural influences on domestic energy consumption. To accomplish this task this paper critically reviews the research methods used in household energy studies, taking into account the pragmatic constraints of different methods of data collection (such as cost and expertise required) and the theoretical constraints (such as type of data collected). In doing so, it provides an understanding of the scope and limitations of both quantitative and qualitative methods of data collection and illustrates how qualitative research methods can be used to expand our knowledge of the social and cultural influences on household energy consumption. This is important, as an understanding of how social and cultural factors shape the technical, economic and behavioural aspects of household energy consumption will enable more accurate assessments of 
both the possibilities for reducing energy consumption in the residential sector and the likely impact of particular energy efficiency interventions.

The remainder of this paper is split into three sections. To contextualise the arguments presented the first section introduces the reader to the role of the social sciences within household energy studies. The second, moves on to critically examine the different methods of data collection utilised in household energy studies, pointing to the possibilities and limitations of each approach to inform our understanding of household energy consumption. It then draws a conclusion as to the scope and limitations of these methods of data collection for uncovering the social and cultural contexts of peoples' lives and how they shape the technical, economic and behavioural factors that influence patterns of household energy consumption. The final section of this paper presents a discussion of the ways in which funding bodies tend to favour quantitative approaches to household energy studies and in so doing reinforce the dominance of those approaches. This section also highlights some innovative research currently being undertaken as part of a longitudinal study of carbon use in buildings, which challenges this trend and gave the author the opportunity to write this paper.

\section{SOCIAL SCIENCE AND HOUSEHOLD ENERGY STUDIES}

Engineers and other natural scientists continue to usefully develop innovative solutions to the question of "how we can be more efficient?" However their work does not answer the question "why are we not more energy-efficient, when clearly it is technically possible for us to be so?" Of course this also includes questioning whether we are developing the right technologies and policies to reduce energy consumption $[9,12,13]$. These types of questions are the realm of the social sciences. For example, research within economics has attempted to address the question of why we are not more energy efficient with reference to market barriers to energy efficiency. The problem with the approach taken by economists is that, on the whole, they assume the choices individuals make with regard to energy consumption are rational, in the sense that they will choose the most efficient option available because it is in their economic interest to do so [14]. However, "[i]t is well known that a lot of energy efficiency improving measures could be realised from a technical point of view and that they would be economically profitable, yet they are neglected"[15 page 833].

Building on the work carried out by economists, researchers within psychology have attempted to account for the how and why people might deviate from expected economic rationality [7 page 52]. The main focus of this work has been attitudes towards energy consumption and saving [16 page 9]. However, it has proved very difficult to predict the linkage between attitudes towards energy saving and actual behaviour and recent psychological research examining home and energy use suggests "that using only attitudinal variables, such as values, may be too limited to explain all types of environmental behaviour" [17 page 70].

In the late 1980s, both sociologists and psychologists began to argue that both rational and attitudinal approaches are severely flawed [7 page 53]. The problem with these approaches is that they both consider energy purchase and use in self-contained individual or household 'units' detached from their socio-cultural context - and thus separated from much that is potentially explanatory [7, 9, 14, 18-21]. For example 
these approaches do not account for "the opportunities and constraints faced by members of various social subgroups [that] have consequences for their work, travel and household composition, and so for everyday energy use practices and stocks of household technology" [14 page 309]. In a response to the critique of attitudinal and rational approaches, to understanding patterns of household energy consumption, 'lifestyle' or culture became the focus of many studies in the late 1990's $[9,16]$.

This shift in focus from attitudes and rational consumers to 'lifestyle,' is argued to have brought about a corresponding shift in research methods: from the quantitative methods traditionally used in the fields of building science, economics and psychology to the more qualitative ethnographic methodologies traditionally used within anthropological and sociological approaches to examining cultural phenomena [16 page 10]. However, the largely quantitative approach traditionally taken in research concerned with energy consumption has led, "perhaps inevitably, to reliance on equally data-led social science research methods which are also largely quantitative and which have attempted to categorise populations into particular cohorts or types" [19 page 2]. This is problematic because the impact of cultural values, which shape the ways in which people consume energy, are being subsumed by the reductive analytical and methodological approaches to energy studies engendered by quantitative methods of data collection. The ways in which this takes place are highlighted in the following section where the scope and limitations of the different methods of data collection used in household energy studies are discussed.

\section{DATA COLLECTION METHODS}

Surveys are the most prevalent method of data collection used in studies exploring household energy consumption. Electronic energy monitoring is also widely used to collect data in household energy studies, while a few researchers have used ethnographic qualitative approaches involving in-depth interviews and focus groups. Each of the methods of data collection used in household energy studies are discussed below, highlighting the advantages and disadvantages of each approach with regard to the possibilities they provide for understanding household energy consumption. Here, both the pragmatic and theoretical constraints of the different methods of data collection are discussed. This is important as the practicalities of using different methods of data collection, (such as cost and technical expertise required) are often as important as theoretical constraints (such as the type of data collected) when designing research strategies. The following section also briefly assesses the use of secondary data in household energy studies. It concludes by summarising the scope and limitations of each of the different methods of data collection for uncovering the social and cultural contexts of people's lives and how they shape the technical, economic, and behavioural factors that influence patterns of household energy consumption.

\subsection{In-depth qualitative interviewing and focus groups}

The flexibility offered by the unstructured format of qualitative interviewing and focus groups often generates two stages of explanatory data. "First is the explanation offered by respondents for their actions in response to direct or indirect questioning. The second comprises synthetic explanations composed by researchers as interviews 
are dissected and analyzed" [8 page 57]. Here what is not said by respondents can be equally as important as their actual replies. For example, a qualitative study of energy decision making in California carried out by Wilk and Wilhite [8] found that solar panels and greenhouses were shown to researchers and discussed with pride by research respondents, that also paraded lower thermostat settings and extra sweaters as stoic adaptations, but these respondents most often passed over or ignored weatherstripping (more commonly known in the UK as draftproofing) [8 page 57]. This omission led to direct questions concerning weatherstripping to be included in the interview schedules, which revealed that inexpensive and efficient technical measures for reducing energy consumption, such as weatherstripping, are often rejected in favour of more expensive, less efficient but more visible technical measures, such as solar power, which have greater social significance [8].

The qualitative study outlined above neatly illustrates that energy consuming behaviours, including the development and diffusion of energy efficient technologies, are a cultural rather than purely economic/ technical phenomenon. In doing so it also demonstrates that the simplistic economic assumptions often drawn in quantitative studies are severely flawed. The findings of Wilk and Wilhite [8] are reinforced by recent qualitative research examining the conversion of electrically heated single family houses to heating by combined pellet stove/solar systems in Scandinavia conducted by Henning [22]. This work found that the home and in particular the fire place has a special place in the heart of many Scandinavian people. As a result of this aesthetically pleasing clean and perhaps expensive pellet stoves are more likely to be accepted in Scandinavians households than less aesthetic looking cheaper models which are dirty and difficult to clean.

Qualitative research has also highlighted the fact that the adoption of energy efficient household technologies does not always result in reduced energy consumption. For example, Naesje [23] examined how heat pumps were used in the domestic environment and found that in some households running hot water was used to cook potatoes, which was not exactly what people were supposed to do to increase the energy efficiency of the household. Thus, this work demonstrates that the efficiency of household technologies not only depends on how they are designed, but also upon how they are used [16]. This perspective can be extended to houses, as there is no guarantee that the occupiers of homes designed to be energy efficient, use those houses as the architects intended. This indicates that energy efficient houses, and household technologies should be designed to fit the lifestyles of householders, rather than expecting householders to change their lifestyles to fit the design of houses and technologies [16, 22].

Cross-cultural qualitative research has demonstrated that some energy consuming practices are more firmly anchored in tradition than others, suggesting that some will be more amenable to change than others [14 page 307]. As mentioned in the introduction research undertaken in Norway and Japan [10] found that in Norway "heating and lighting have important symbolic value [being used] in combination to create what the Norwegians call cosiness, a state of comfort that is practically mandatory for Norwegian living rooms.............. [and that] the strong social significance of cosiness leads to overheating and over lighting as insurance against 
social failure" [10 page 798]. In Japan aesthetic considerations were found to be no less significant than in Norway, but they took a very different form. As a result Japanese living rooms are exclusively lit by central ceiling lamps, brightness is preferred and fluorescent lighting is the norm. However, the Japanese traditional form of bathing, which involves combining showering and taking long heated baths, is heavily energy intensive, whereas in Norway showering was found to be the norm and therefore Norwegian bathing habits are much less energy intensive than those of the Japanese.

Of course in-depth interviews and focus groups are susceptible to self-report bias. This problem was highlighted by recent work using in-depth interviews and energy modelling for communicating about energy use and revealing consumer preferences for change at different levels and intervention points [24]. This study found that research participants generally overestimated the environmental friendliness of their lifestyles. The research subjects were interviewed both before, and after their consumption patterns were modelled using a sophisticated computer program. The findings from this process illustrated that these individuals used considerably more energy in their everyday lives than they had believed they used prior to the modelling process.

Focus groups and in-depth interviewing are usually more expensive than survey based research. Typically in-depth interviews and focus groups are electronically recorded adding transcription costs to the research process. Added to this, in the data collection phase of the research, qualitative studies demand more skilled and therefore more expensive researchers than is the case for survey based research. Survey based interviews are often merely a case of reading a set of predefined questions and noting down which of the predefined answers the respondent selected. Whereas, in-depth interviews and focus groups demand that the researcher conducting them has both the background knowledge and interview skills to obtain the required information from research participants. The probing nature of the questions asked in in-depth interviews and focus groups are also more invasive and time consuming than the closed type of questions asked in survey based research. Thus, focus groups and in-depth interviews are normally more time consuming for both the researcher and the research participant, adding to both their financial cost and the level of participant commitment necessary to conduct the research, when compared to survey based methods.

The difficulties of conducting in-depth interviews and focus groups and their cost are not seen as the main limitation of these styles of data collection. The main difficulty with these approaches is perceived to be that they do not lend themselves to statistical analysis. The use of open-ended questions means that it is difficult to quantify responses and the time and expertise necessary for conducting both focus groups and in-depth interviews usually restricts the numbers of participants, to those well below the number necessary for producing valid statistical results. However although in-depth interviews and focus groups "do not usually produce statistically valid results that can be freely generalized to the larger population of energy users, they do yield finely grained and detailed information that cannot be obtained through questionnaires, and they often provide unexpected insights and lead to productive new lines of inquiry [8 page 52]. 
In summary, qualitative methods of data collection have the advantage that they provide the depth of data necessary for an analysis of the social and cultural factors that underpin energy consuming behaviour. However, they also have the disadvantage that they are susceptible to self-report bias, expensive and do not lend themselves to statistical analysis.

\subsection{Monitoring household energy consumption}

Rather than relying on research participants to give accurate estimates of how much energy they consume, energy monitoring studies supply data based on actual energy consumption [see refs 25-37]. This is significant when considering the social and cultural aspects of peoples' energy consuming choices, as the inaccuracies inherent in the self-reporting of energy consumption can lead to the underestimation of the relationship between behaviour and energy consumption [38 page 1047]. In contrast to studies that rely on the self-reporting of energy consumption, those measuring energy consumption have repeatedly shown that everyday energy consuming behaviours are significant to overall consumption levels. For example, early energy monitoring studies found that nearly identical housing, occupied by families of similar composition can have variations in energy consumption patterns of between two and three hundred percent [31, 32].

Studies which monitor the energy consumption of individual appliances can provide detailed data on the amounts of energy consumed by every day practices such as heating, washing, bathing, cooking and lighting. This can allow a fine-grained analysis of how factors such as, household composition, the use of energy efficient technologies and the style, age and insulation levels of residential properties effect domestic energy consumption. For example, energy monitoring studies have shown that energy consumption levels vary according to the number of people living in households, the age and economic status of residents and their working patterns [26, 30]. The data provided by energy monitoring studies can be used to illustrate which daily practices use the most energy, or to indicate where there are large discrepancies in the amounts of energy used by different types of households for the same daily activities. In doing so energy monitoring studies offer the potential to provide valuable insights into which daily household energy consuming activities might be most influenced by social and cultural factors, and where energy saving initiatives should be focused to have the greatest impact.

The detailed data on the energy consumed by everyday practices made available by energy monitoring studies also presents the opportunity to assess interventions designed to reduce household energy consumption. A recent example examined the application of information-feedback methods for saving energy and found that regular feedback using electronic methods produced larger energy savings, than merely providing paper based information to householders [28]. However this study, as with many of its predecessors, took an instrumental approach. Its concern is with "how we can reduce energy-consumption with existing end-use equipment in the residential sector, rather than why socio-cultural population demographics currently causes substantial differences in rates of energy use” [28] [page 823]. The position taken here is indicative of how social and cultural differences are subsumed by the dominant 
styles of analysis and data collection within household energy research. This approach neglects questions such as why those that took part in the study reacted most positively to electronic energy consumption feedback. This omission of course leaves the question of whether, under all circumstances, electronic feedback will have a more positive effect on levels of energy consumption than paper based methods unanswered.

The discussion above points to the major limitation of energy monitoring studies; which is that, unless they are combined with other methods of data collection, they provide no information on the human dimensions of energy consumption. To offer another example, it has been shown that there is up to a fifty percent variation in the electricity used to cook the same meal with the same equipment by different chefs [39], however we cannot know why this occurred without speaking to the chefs in question. Thus, while energy studies can illustrate disparities in how much energy is used by the daily actions of different groups of householders, they can not, on their own, provide information concerning why this might be the case. However, the very fact that energy monitoring studies have illustrated that nearly identical housing, occupied by families of similar compositions, can have large variations in energy usage $[31,32]$ points to the importance of cultural and social influences on energy consumption.

The benefits of energy monitoring over survey research have long been understood. Writing in 1986 Vine [40 page 122] claimed that "ideally one would like to monitor the indoor temperatures of residential households to determine if people are adjusting their thermostats to reduce energy use. Interestingly, in spite of this recognition, there are few energy monitoring studies in comparison to studies using survey methods to record energy consumption. This is probably partly due to the fact that the technology and expertise necessary for monitoring energy consumption can be prohibitively expensive [40 page 122]. It has been argued that energy monitoring "is usually not practical when a large sample size is targeted" [41 page 1680] ${ }^{1}$.

The cost of energy monitoring studies is further increased by the need for the data to be collected over long time periods. This is because monitoring energy consumption may cause research participants to deviate from their normal energy consuming behaviours. Thus, the time over which the data is recorded must be long enough to allow participants to revert to their normal behaviours. Measuring household energy consumption is also an invasive process and therefore necessitates a relatively large commitment from research participants. This can make it difficult to get people to take part in energy monitoring studies [31 page 204].

In summary, energy-monitoring studies have major advantages and disadvantages when compared to the other methods of data collection used in household energy studies. The advantages being that they provide information on actual consumption patterns rather than having to rely on self reported consumption and they can provide detailed data on the amounts of energy consumed by everyday practices such as heating, washing, bathing and cooking. However, energy monitoring is expensive, time consuming and unless combined with complementary methods of data collection

\footnotetext{
1 Advances in metering technologies in some countries, can significantly reduce the cost of energy monitoring studies. See for example Pratt et al. [34] and Parker [42].
} 
it does not provide any data on the social and cultural aspects of peoples lifestyles which create many of the disparities in household consumption patterns. In household energy studies where energy monitoring has been combined with other data collection methods, these have been predominantly survey-based methods, the scope and limitations of which for understanding the social and cultural aspects of energy consumption are discussed below.

\subsection{Surveys of householders}

Most of the social science research exploring household energy consumption has used some form of survey based method of data collection. The closed question format, used in both self-report and administered surveys, facilitates the collection of easily quantifiable data from large populations, enabling statistical examinations of the influence of different variables on household energy consumption patterns and behaviours. Many studies into household energy consumption have used survey based research to explore the relationship between socio demographic and/or technical factors, and energy consumption patterns and behaviours. In this way work in this area has pointed to the possible relationships between factors such as home ownership, income, education and energy consuming behaviours [see 43-45]. A classic example is provided by Black et al [45]. This study identified a strong correlation between homeownership and large capital investment in energy saving measures. It also found that there was no correlation between homeownership and the adoption of energy efficient behavioural changes such as switching of lights in unused rooms or always putting a full load in the washing machine. However, these findings tell us very little about why homeowners were prepared to make large capital investments in energy efficiency technologies and nothing about the reasons why people adopt, or do not adopt, energy efficient behaviours.

The move in the 1990's toward focusing household energy studies on 'lifestyles' and culture brought about a large number of survey based studies in which 'lifestyles' are seen as recipes for living, that differentiate social groups from one another [19]. Studies of this type are often also based primarily on demographic factors (age, ethnicity, household composition and so on). However, many socio-demographic factors are correlated with house form, appliance holdings, and energy consumption levels, and can therefore offer crude indications of cultural networks [7 page 56] $]^{2}$. It is also possible to combine demographic data with other lifestyle indicators in surveybased research in order to refine socio-demographic categorisations of consumers. For example, through a survey registering socio-economic aspects, housing standards, attitudes and media usage among other issues Ljones and Doorman [47] (quoted from Aune [16] page 16) constructed seven energy cultures: the value seeker, the life style simplifier, the nest builder, the trouble avoider, the resource conscious, the toiler and the idealist.

However, while survey based lifestyle research can be used to identify different sub-groups within society based on their consumption patterns, they do not provide the

\footnotetext{
2 This position is backed up by recent survey based work [46] carried out in 2002 which indicates that household energy use is strongly related to variables such as household size and income.
} 
information to explore the reasons why the persons within these subgroups act and choose in the ways that they do. Therefore, lifestyle focused surveys like the earlier socio-demographic surveys do not answer the question "why are we not more energyefficient, when clearly it is technically possible for us to be so?" This is in part due to the limitations of surveys as a method of data collection. As pointed out by Wilhite et al "an analysis and interpretation of complex culture based household behaviours........ is very difficult to achieve in a closed format interview or from survey questionnaire responses" [10 page 796]. The inability of surveys to answer 'why' questions often leads to the reasons for particular behaviours being inferred from correlations between the variables under study. For example, Poortinga et al [48] found a positive correlation between higher incomes and the acceptability of technical efficiency measures, and the authors suggest that this "might be explained by the fact that technical measures often require an initial investment, which might be less problematic for the higher-income groups" [48 page 60]. However, this conclusion is mere speculation, as the research supplied no evidence to support this argument.

The preference for survey research within household energy studies is probably due, in part, to the relatively low cost of carrying out surveys. It is the cheapest method of obtaining data from populations large enough to allow the development of valid statistical generalisations and is "still believed to be one of the most cost-effective approaches to measure consumed energy by end-uses" [41 page 1680]. This is particularly the case with self-report surveys which do not involve the expense of employing researchers to administer the survey. However, in many cases, the extra cost of using administered surveys can be justified because they have the advantage that incomplete or obscure answers can be clarified by the researcher conducting the interview improving the quality of the data obtained when compared to self-report surveys. There are also some differences in the levels of participant comment necessary for administered and self-report surveys. It takes relatively little time to fill in a questionnaire which can be completed at the respondent's convenience, whereas finding the time to be interviewed demands more of a commitment from research participants. However, both types of surveys can be limited by low response rates [41 page 1681].

Survey based research is also limited by its reliance on the self-reporting of energy consumption patterns and behaviours. The issue of self-report bias is particularly problematic with studies attempting to use survey research to estimate energy consumption, rather than energy consumption behaviours or end uses. For example, it is much easier to recall whether or not you leave lights on in unoccupied rooms or what temperature you set your thermostat in winter and summer, than to recall the number of gas or electricity units you used in the last quarter.

In summary, as with the other methods of data collection discussed here, survey based approaches have major advantages and disadvantages when used in household energy studies. The main advantage is that they are highly amenable to statistical analysis. The use of closed questions facilitates the quantification of the data collected, while the low cost of surveys enables data to be gathered from populations large enough for statistical generalisations to be drawn. As is the case with qualitative 
methods of data collection surveys are restricted by their reliance on self-reports. Surveys are also often limited by low response rates. However the main limitation of survey approaches to data collection is that they do not lend themselves to the use of open ended questions and therefore it is difficult to use them to explore the social structures and cultural expectations which underpin the reasons why different social groups consume different amounts of energy. Thus, while survey based work can provide a statistical method of identifying different subgroups within society based on their consumption patterns, it can not tell us why the individuals within these subgroups act and choose in the ways that they do, or what opportunities and constraints they face in making those choices.

\subsection{Analysis of secondary data}

"The analysis of secondary data involves the utilisation of existing data, collected for the purposes of a prior study, in order to pursue a research interest which is distinct from that of the original work" [50 para. 1].

As discussed earlier, despite calls for more research within the qualitative tradition in the 1980's and 1990's, far fewer studies exploring household energy consumption have been conducted using in-depth interviews and/or focus groups than have been conducted using quantitative surveys or energy monitoring approaches to data collection. Therefore, there is very little qualitative data available for secondary analysis. This problem is exacerbated by the fact that re-use of qualitative data remains theoretically and methodologically under-developed [50].

On the other hand, the re-analysis of statistical data is an established and welldocumented methodology and there is plenty of data available from a myriad of sources including utility companies, large government surveys of consumption and previous work into household energy consumption. If exploring variation in the behaviours/ attitudes of population subgroups (e.g. elderly, disabled, socioeconomically disadvantaged), by geography (e.g. region, urban/rural, country) the reuse of data from large government funded surveys is often the only method of gaining statically valid samples. The use of government surveys which are repeated regularly, such as the 'British Social Attitudes' Survey, can also enable longitudinal analysis. The use of this type of survey is often the only method of obtaining data which allows for the statistical comparison of attitudes over time.

The analysis of secondary quantitative data can illustrate the importance of social and cultural influences on household energy consumption. As demonstrated by a recent cross cultural quantitative analysis of household energy consumption patterns in Australia, Brazil, Denmark, India and Japan. This study used data from government expenditure surveys and other sources. It indicated that "characteristics of energy consumption are unique to each country, and determined by distinctive features such as resource endowment, historical events (such as energy supply shortages or introduction of taxes), socio-cultural norms, behaviour and present market conditions, as well as energy and environmental policy measures" [51 page 201]. The authors conclude that their findings "indicate there is no one-fits-all recipe for planning for energy reductions" [51 page 201]. However, what this research does not tell us is how particular socio-cultural norms in specific contexts shape energy consuming practices, 
for this we need more in-depth information that can only be gained from more qualitative approaches.

Despite the differences between the secondary analysis of qualitative and quantitative data some of the difficulties involved are similar. One of the main problems encountered by Elkins et al [52] in their quantitative household energy study was "the lack of timeliness of even the most up to date published statistics" [52 page 49]. This is also a problem for researchers attempting to analyse secondary qualitative data. An early qualitative study of energy behaviour conducted in 1984 found that Norwegian householders [53] were more likely to trust energy companies to implement energy efficiency measures in their homes than American householders. However, we can not rely on attitudes to energy companies being the same today as they were over twenty years ago. Analysing data from studies designed to ask different questions from those currently under examination is also problematic, regardless of whether the researcher is attempting to re-analyse qualitative or quantitative data. This type of problem is highlighted by Vine's qualitative study of thermostat settings [40], he found that existing data was not of good enough quality to support or reject his hypotheses, and due to the diverse nature of the sources on offer it was difficult to synthesize the data.

In summary, in the case of qualitative studies the re-analysis of secondary data has the advantage that large data sets are available from government and other sources. These are often much larger than it would be possible for researchers to obtain by any other method and in some cases offer opportunities for longitudinal analysis. However, as discussed throughout this paper, quantitative data does not provide the depth of data necessary for examining the complex socio-cultural aspects of household energy consumption. Therefore, the reanalysis of secondary quantitative data will not provide us with the information with which to understand why people make the energy consuming choices that they do. The re-analysis of secondary data is also limited by the availability of relevant timely data, which is particularly problematic in the case of qualitative data. Even where data is available, there are problems created by the design and execution of the original study which collected the data. Once again, this is particularly problematic in the case of qualitative data because the re-use of qualitative data remains theoretically and methodologically under-developed [50].

\subsection{The most promising methodological approaches}

What, then, are the most promising methodological approaches for exploring the interaction between the social and cultural aspects of peoples' lives and the technical, economic and behavioural factors that influence levels household energy consumption? Table A, provides a summary of the scope and limitations of each of the methods of data collection used in household energy studies in this regard, and clearly illustrates that qualitative methods of data collection are the road forward. 


\section{Table A}

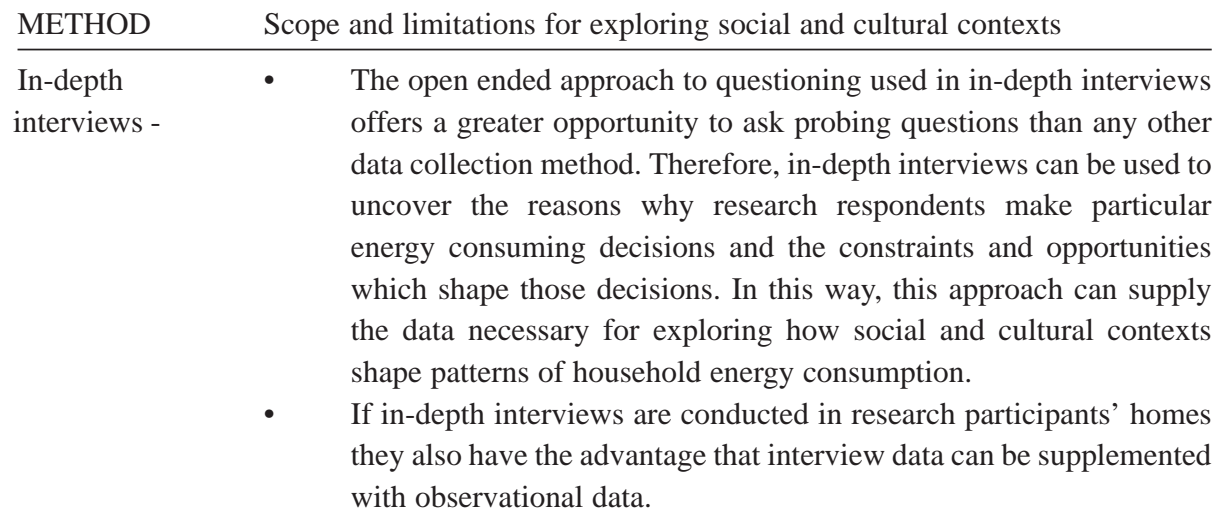

Focus groups - $\quad$ Focus groups offer the opportunity to listen as people share and compare their different points of views. This provides information concerning not only what people think and how they act, but also why they think and act in the way they do. Therefore, this approach can provide the depth of data necessary to exploring the social and cultural aspects of household energy consumption, because it allows an exploration of the opportunities and constraints faced by research participants in their everyday energy consuming choices.

Monitoring - Unless combined with other methods of data collection this energy consumption approach provides no information on why people make particular energy consuming choices and decisions. Therefore monitoring energy consumption on its own can not supply the data necessary for exploring social and cultural aspects of domestic energy consumption.

Self -report $\quad$ - Because self-report surveys employ closed question formats it is Surveys extremely difficult to use this approach to uncover the reasons for the energy consuming choices made by research respondents. For example, they can be used to obtain information concerning how many people in a given population own particular household appliances, but not why research respondents choose to buy or not to buy particular appliances. Therefore this approach supplies very little data on how social and cultural contexts shape household energy consumption.

Administered - The closed question format of administered surveys severely restricts surveys / probing questions. Thus, as with self-report surveys it is difficult to structured address questions concerning the social mores and cultural interviews expectations which underpin the reasons why different groups 
Secondary analysis of data- consume different amounts of energy.

- The closed format of administered surveys can also restrict the recording of supplementary observational data when interviews are conducted in research participants' homes.

- The qualitative data-sets available for secondary analysis are limited and quantitative data sets do not provide the necessary depth of data for exploring social and cultural factors

- Data quality is totally dependent on the methodology applied in the original research.

- The re-use of qualitative data remains theoretically and methodologically under-developed

- $\quad$ Typically secondary analysis of data is exploring different questions to those for which the data was gathered in the original study. This often means that there are gaps in the data in regard of the questions being asked by the secondary analysis.

However, as mentioned earlier, this is not to suggest that quantitative methods have no place in energy studies. The author suggests the monitoring of energy consumption is a necessity within household energy studies, as only this method avoids the issue of self-report bias. Survey based research and secondary data analysis also have a place in household energy studies, as these approaches can provide a wealth of information on the different demographic and technical factors effecting household energy consumption. However, if we are to inform initiatives designed to encourage the adoption and use of energy efficient technologies in the home, quantitative methods of data collection must be integrated with more qualitative ethnographic research: as only qualitative methods of data collection provide the depth of data necessary for exploring how the social and cultural aspects of peoples' lives shape the technical, economic and behavioural factors that influence levels household energy consumption. Therefore, it is only studies with a substantial qualitative element that can inform the introduction of technically proved energy efficient technologies into appropriate social practices.

\section{RESEARCH FOCUS, METHODS OF DATA COLLECTION AND FUNDING OPPORTUNITIES}

Many people enjoy heavily energy intensive practices, such as lounging in hot tubs and power showers and having large numbers of lighting sources to create different lighting moods. As a result of this enjoyment people are often very reluctant to reduce the amount of energy they consume. Raising the cost of energy to restrict household energy consumption is not an option given the problems this would create for those at the lower end of the economic spectrum. It must also be noted that increasing the cost of energy has the least effect on the households that consume largest amounts of energy; as they tend to be wealthy households that can easily afford price increases. This alone indicates that more thought, time and money must be invested in researching peoples' energy consuming practices and designing energy efficient 
technologies to fit those practices if the take up of energy efficient technologies is to increase.

However, If we are to begin to understand the complex socio-cultural nature of household energy consuming practices we can not merely tack some qualitative interviews or focus groups on to the end of quantitative research strategies; rather, they must be central to the research perspective taken. As discussed throughout this paper, the skills needed to collect and analyse qualitative data are different from those involved in quantitative research. However, it is not merely a case of researchers within household energy studies accepting the validity of qualitative methods and learning how to apply them. Those involved in the funding of energy research must also embrace the validity and findings of qualitative research.

The challenge of improving the energy efficiency of buildings has been constructed as a task for building science [1]. The traditional role of the social sciences in this endeavour has largely been limited to quantitative economic and psychological research into the barriers to energy efficiency [54]. "The institutions of research funding together, with [these] conventions of research practice have hooked up with a model of energy policy focused on the production of transferable knowledge and the analysis of non-technical barriers to its effective implementation"[1 page 65]. Thus, as qualitative studies do not produce statistically valid results, enabling the claim that research findings can be freely generalised to the larger population of energy users, they are unpopular with both the statutory and non-statutory bodies that fund household energy research. This is, in part, because funding organisations naturally desire the ability to inform policies and interventions aimed at reducing household energy consumption that can be re-used to produce the same results in many different contexts. However, as discussed earlier, culturally sensitive research has illustrated that energy consuming behaviours, including the development and diffusion of energy efficient technologies, are social and cultural phenomena rather than being purely technical or economic issues. This suggests the search for fully generalisable solutions to decreasing household energy consumption that can be freely generalised to all household energy users is over ambitious.

\subsection{Carbon Reduction in Buildings (CaRB)}

As discussed throughout this paper, there is a growing body of research which illustrates that technical and economic measures for reducing energy demand need to be culturally specific and the 'one size fits all' mentality must be changed if we are to make any progress in reducing energy demand in contemporary society. Some funding bodies have embraced these findings and are funding research which seeks to combine both qualitative and quantitative methods. One such piece of work is currently being conducted by the Carbon Reduction in Buildings (CaRB) consortium, under the Carbon Vision programme.

$\mathrm{CaRB}$ recognises that reducing carbon emissions from buildings requires understanding both the technical and social dimensions of energy use. Toward this end part of the CaRB team are examining the social and cultural influences on home energy use. This research will use qualitative and quantitative methods to explore, the 
competing interests involved in the design and marketing of technologies, how people use technologies in practice, how these practices are affected by the design of technologies, and how this affects home energy use. By exploring the competing interests involved in the design of technologies this work embraces the notion that individuals energy consuming choices are shaped by the decisions made by individuals within many organisations. The $\mathrm{CaRB}$ team are also monitoring household energy consumption so that the research is grounded in measured energy consumption, rather than estimations.

\section{ACKNOWLEDGMENTS}

The author thanks Professor Simon Guy and Dr Susannah Gun for their helpful and insightful comments on earlier drafts of this paper.

\section{REFERENCES}

1. Guy, S., Shove, E., A Sociology of Energy Buildings and the Environment, London, Routledge, 2000.

2. Parker, P., Scott, D., and Rowlands I. H., Residential Energy Consumption and Greenhouse Gas Emissions: Global Context for Local Action, Environments: 2001, 28(3):11-28.

3. Environment Agency, Environmental Facts and Figures: Households, 2005, http://www.environmentagency.gov.uk

4. Australian-Bureau-of-Statistics, Australian Social Trends 1998. People \& the Environment - Use of Resources: Household energy use. 2002. http://www.abs.gov.au/Ausstats/abs@.nsf/Lookup/1DDF10C29E1C558ACA2569AD0004 02D6

5. UNESCAP, Guidebook on Promotion of Sustainable Energy Consumption, 2001, http://www.unescap.org/esd/energy/publications/psec/index.htm

6. Tso, G. K. F., Yau, K. K. W., A study of domestic energy usage patterns in Hong Kong, Energy, 2003, 28(15):1671-1682.

7. Lutzenhiser, L., A cultural model of household energy consumption, Energy, 1992, 17(1), 47-60.

8. Wilk, R., Wilhite, H., Why Don't People Weatherize Their Homes? An Ethnographic Solution, in: Energy Efficiency: Perspectives on Individual Behavior, edited by Kempton W, Neima M., Washington, DC and Berkeley, CA: American Association for an Energy Efficient Economy; 1986: 51-68.

9. Guy, S., Consumption, Energy, and the Environment, Encyclopaedia of Energy, 2004, 1:687-695.

10. Wilhite, H., Nakagami, H., Masuda, T., Yamaga, Y., Haneda, H., A cross-cultural analysis of household energy use behaviour in Japan and Norway, Energy Policy, 1996, 24(9), 795803.

11. Owens, J., Wilhite, H., Household energy behavior in Nordic countries-an unrealized energy saving potential, Energy, 1988, 13(12), 853-859. 
12. Shove, E., Wilhite, H., Energy policy: what it forgot and what it might yet recognise, in: ECEEE Summer Study Proceedings: May 31 - June 4 1999, Mandelieu, France.

13. Chappells, H., Shove, E., Debating the future of comfort: environmental sustainability, energy consumption and the indoor environment, Building Research and Information, 2005, 33(1), 32-40.

14. Shove, E., Lutzenhiser, L., Guy, S., Hackett, B., and Wilhite, H., Energy and Social Systems, in: Human Choice and Climate Change: Resources and Technology, edited by Rayner S, Malone E, vol. 2. Columbus Ohio, Battelle Press; 1998, 291 - 325.

15. Weber, L., Some reflections on barriers to the efficient use of energy, Energy Policy, 1997, 25(10), 833-835.

16. Aune, M., Thomas, B., Sorensen, K., Needs, Roles and Participation: A review of the social science studies of users in technological design, A report within the research program Smart Energy-Efficient Buildings at NTNU and SINTEF 2002-2006, 2002.

17. Poortinga, W., Steg, L., and Vlek, C., Values, Environmental Concern, and Environmental Behavior: A Study into Household Energy Use, Environment and Behavior, 2004, 36(1),7093.

18. Fujii, H., Lutzenhiser, L., Japanese residential air-conditioning: natural cooling and intelligent systems, Energy and Buildings 1992, 18(3-4), 221-233.

19. Strang, V., Contextualising Values and Behaviour in: Proceedings of the European Council for an Energy-Efficient Economy, June 1977.

http://www.eci.ox.ac.uk/lowercf/pdfdownloads/ECEEE97_VS.pdf

20. Wilhite, H., What can energy policy learn from thinking about sex, in: Summer Study Proceedings European Council for an Energy-Efficient Economy 2001.

21. Wilk, R., Consumption, human needs, and global environmental change, Global Environmental Change 2002, 12(1), 5-13.

22. Henning, A., Social anthropological and interdisciplinary research on the conversion of electrically heated single family houses to heating by combined pellet-solar systems. Biomass and Bioenergy 2004, 27(6):547-555.

23. Naesje, P., Pump and circumstances. The political configuration of heat pump technology in Norway, STS report, 2000, NTNU, Department of interdisciplinary studies of culture.

24. Goldblatt, D. L., Hartmann, C., Durrenberger, G., Combining interviewing and modeling for end-user energy conservation, Energy Policy, 2005, 33(2),257 - 271.

25. Harrje, D. T., Grot, R. A., Instrumentation for monitoring energy usage in buildings at Twin Rivers, Energy and Buildings 1978, 1(3),293-299.

26. Andrade, J. A., The uses of energy in the domestic sector, Energy and Buildings 2001, 33(6), 525-529.

27. Mansouri, I., Newborough, M., Dynamics of energy use in the UK households: end-use monitoring of electric cookers, In: European Council for an Energy-Efficient Economy, Summer Study Proceedings: 1999. 
28. Wood, G., Newborough, M., Dynamic energy-consumption indicators for domestic appliances: environment, behaviour and design, Energy and Buildings 2003, 35(8), 821841.

29. Ueno, T., Sano, S., Saeki, O. and Tsuji, K., Effectiveness of an energy-consumption information system on energy savings in residential houses based on monitored data, Applied Energy 2006, 83(2), 166-183.

30. Tribwell, L., Shedding Light On Home Lighting Use, Home Energy 1997, 14(1), 15-19.

31. Socolow, R. H., Sonderegger, R. C., The Twin Rivers Program on Energy conservation in Housing: four Year Summary Report, No 32, Princeton, NJ, Princeton University, Energy Environment Study, 1976.

32. Sonderegger, R. C., Movers and stayers: The resident's contribution to variation across houses in energy consumption for space heating, Energy and Buildings 1978, 1(3),13-24.

33. Parker, D. S., Research Highlights from a Large Scale Residential Monitoring Study in a Hot Climate in: Proceedings of International Symposium on Highly Efficient Use of Energy and Reduction of its Environmental Impact, Japan Society for the Promotion of Science Research for the Future Program, JPS-RFTF97P01002, Osaka, Japan, January, 2002, 108116.

34. Pratt, R. G., Description of Electric Energy Use in Single-Family Residences in the Pacific Northwest, in: End-Use Load and Consumer Assessment Program (ELCAP), Pacific Northwest Laboratory, DOE/BP-13795-21, Richland, WA,; 1989.

35. Fuentes, M., Dichler, A., Roaf, S., The Oxford Solar House, Renewable Energy 1996, 8(14):145-153.

36. Oreszczyn, T., Hong, S. H., Ridley, I., Wilkinson, P., Determinants of winter indoor temperatures in low income households in England, Energy and Buildings, In Press, Corrected Proof.

37. Hunt, D. R. G., Gidman, M. I., A national field survey of house temperatures, Building and Environment, 1982, 17(2), 107-124.

38. Mullaly, C., Home energy use behaviour: a necessary component of successful local government home energy conservation (LGHEC) programs, Energy Policy 1998, 26(14), 1041-1052.

39. Fechner, J. V., Human factors in appliance energy-consumption, in: Proceedings of the IEEE Appliance Technical Conference, Pittsburgh, Pennsylvania, 1977.

40. Vine, E. L., Saving energy the easy way: An analysis of thermostat management. Energy, $1986,11(8), 811-820$.

41. Tso, G., and Yau, K., A study of domestic energy usage patterns in Hong Kong. Energy, 2003, 28(15), 1671-1682.

42. Parker, D. S., Research Highlights from a Large Scale Residential Monitoring Study in a Hot Climate, in: Proceedings of International Symposium on Highly Efficient Use of Energy and Reduction of its Environmental Impact, Japan Society for the Promotion of Science Research for the Future Program, JPS-RFTF97P01002, Osaka, Japan, January, 2002: 108-116. 
43. Painter, J., Semenik, R., Belk, R., Is there a generalized energy conservation ethic? A comparison of the determinants of gasoline and home heating energy conservation, Journal of Economic Psychology, 1983, 3(3-4), 317-331.

44. Dillman, D. A., Rosa, E. A., Dillman, J. J., Lifestyle and home energy conservation in the United States: the poor accept lifestyle cutbacks while the wealthy invest in conservation, Journal of Economic Psychology, 1983, 3(3-4), 299-315.

45. Black, J., S., Stern, P., C., and Elworth, J., T., Personal and contextual influences on household energy adaptations, Journal of Applied Psychology 1985, 70(1), 3-21.

46. Gatersleben, B., Steg, L., and Vlek, C., Measurement and Determinants of Environmentally Significant Consumer Behavior, Environment and Behavior 2002, 34(3):335-362.

47. Ljones, A., Doorman, G., Energy marked survey 1991 ED92-196, Energidata, Trondheim 1992 (Energimarkedsunders $\emptyset$ kelsen 1991- only available in Norwegian)

48. Poortinga, W., Steg, L., Vlek, C., and Wiersmac, G., Household preferences for energysaving measures: A conjoint analysis. Journal of Economic Psychology 2003, 24(1):49-64.

49. Warriner, G. K., McDougall, G. H., Claxton, J. D., Any data or none at all? Living with inaccuracies in self-reports of residential energy consumption. Environment \& Behavior 1984, 16(4):503-526.

50. Heaton, J., Secondary analysis of qualitative data. Social Research Update, Department of Sociology, University of Surrey, Guildford GU2 7XH, England 1988, 22. http://www.soc.surrey.ac.uk/sru/SRU22.html

51. Lenzen, M., Wier, M., Cohen, C., Hayami, H., Pachauri, S., Schaeffer, R., A comparative multivariate analysis of household energy requirements in Australia, Brazil, Denmark, India and Japan, Energy, 2006, 31(2-3), 181-207.

52. Ekins, P., Russell, A., and Hargreaves, C., Reducing carbon emissions through improved household energy efficiency in the UK, Journal of Environmental Policy \& Planning, 2002, 4(1): $41-65$.

53. Wilhite, H., Improving the ENØK-Consultants interaction with Residential Clients, GRS597 Resource Policy group, 0458 Oslo 4, Norway, 1984.

54. Shove, E., Gaps, barriers and conceptual chasms: theories of technology transfer and energy in buildings, Energy Policy 1998, 26(15), 1105 - 1112.

55. Lutzenhiser, L., Social and Behavioural Aspects of Energy Use, Annual Review Energy and Environment , 1993, 18, 247 -289. 
